\title{
Article \\ Older Drivers' Motion and Simulator Sickness before and after Automated Vehicle Exposure
}

\author{
Sherrilene Classen ${ }^{1}$, Seung Woo Hwangbo ${ }^{1, * \mathbb{D}}$, Justin Mason ${ }^{1}{ }^{\mathbb{D}}$, James Wersal ${ }^{1}\left(\mathbb{D}\right.$, Jason Rogers ${ }^{1}(\mathbb{D}$ \\ and Virginia P. Sisiopiku 2 (I) \\ 1 Department of Occupational Therapy, University of Florida, Gainesville, FL 32611, USA; \\ sclassen@phhp.ufl.edu (S.C.); justinmason@phhp.ufl.edu (J.M.); jwersal@phhp.ufl.edu (J.W.); \\ jhodge@phhp.ufl.edu (J.R.) \\ 2 Department of Civil, Construction, and Environmental Engineering, University of Alabama at Birmingham, \\ Birmingham, AL 35294, USA; vsisiopi@uab.edu \\ * Correspondence: shwangbo@phhp.ufl.edu; Tel.: +1-352-273-6043
}

Citation: Classen, S.; Hwangbo, S.W.; Mason, J.; Wersal, J.; Rogers, J.; Sisiopiku, V.P. Older Drivers' Motion and Simulator Sickness before and after Automated Vehicle Exposure. Safety 2021, 7, 26. https://doi.org/ $10.3390 /$ safety7020026

Academic Editors: Martin Lavallière and Garrett Mattos

Received: 19 February 2021

Accepted: 26 March 2021

Published: 2 April 2021

Publisher's Note: MDPI stays neutral with regard to jurisdictional claims in published maps and institutional affiliations.

Copyright: (c) 2021 by the authors. Licensee MDPI, Basel, Switzerland. This article is an open access article distributed under the terms and conditions of the Creative Commons Attribution (CC BY) license (https:// creativecommons.org/licenses/by/ $4.0 /)$.

\begin{abstract}
Older drivers desire independence in mobility, and automated vehicles hold plausible opportunities to realize this goal. Motion sickness (automated shuttle exposure) or simulator sickness (automated driving simulator exposure) may affect acceptance of these technologies. This study investigated the onset of motion and simulator sickness in older drivers (mean age $=74.29$, $S D=5.96$; female $=54 \%$ ) after exposure to an automated shuttle and automated driving simulator and assessed age and sex as determinants of motion and/or simulator sickness. Using a repeated measures design, 104 older drivers were randomly allocated to the shuttle and simulator. Baseline, as well as post exposures, were measured using the Motion Sickness Assessment Questionnaire (domains: sweatiness, queasiness, dizziness, nauseousness). Older drivers who were exposed to the simulator show a statistically significant increase in simulator sickness symptoms across the four domains compared to the same group being tested in the shuttle. No age and sex differences were detected within the groups and no participants dropped out of the study due to motion or simulator sickness. The automated shuttle and simulator hold plausible opportunities for continued exposure of older drivers to these technologies, as long as motion or driving simulator sickness protocols are used properly.
\end{abstract}

Keywords: older drivers; automated shuttle; motion sickness; automated driving simulator sickness

\section{Introduction}

The deployment of automated vehicles (AV) may offer numerous safety benefits for older drivers, i.e., potentially reducing crash risks, keeping older drivers on the road longer and safer, or being actively engaged in their communities through automated ridesharing services [1]. However, AVs may provoke motion sickness [2] with signs and symptoms including nausea and vomiting [3]. Automated driving simulation is an invaluable tool for exposing older drivers to AVs since it reduces risks associated with using such modes on the road. Simulator sickness, however, is associated with virtual environments [4] and includes symptoms such as sweating, nausea, vomiting, and dizziness [5]. In earlier studies, susceptibility to motion sickness and driving simulator sickness has been linked to sex, age, previous motion sickness, health conditions, and/or medications [6-8]. Females and older adults, especially the young-old (i.e., 65-74 years) and old-old (i.e., $\geq 75$ years), are more susceptible to motion sickness [9] and simulator sickness during driving simulation [7,10-12]. However, it is unclear if these effects persist while older adults are riding in an AV; yet, we expect that simulator sickness will occur if older drivers are exposed to a driving simulator running in automated mode. 


\subsection{Older Drivers}

Driving is the preferred mode of transportation for elderly and enables them to maintain their independence, autonomy, identity, social roles, and well-being $[13,14]$. Driving cessation has negative effects for older drivers such as depression and/or decreased physical activity levels [15-17]. As such, AVs provide plausible opportunities to examine the potential for continued lifelong mobility.

\subsection{Automated Vehicles}

Fully AVs [18] (Society of Automotive Engineers; SAE, Level 4-5) may prevent car crashes, reduce heavy traffic congestion, and reduce gas emissions [19] if they are widely accepted and adopted by the public. Because older drivers are at a high risk for fatalities due to motor vehicle crashes, $\mathrm{AVs}$ hold numerous safety benefits for this population $[1,20,21]$. However, little is known about older drivers' motion or simulator sickness while riding in an automated shuttle (Level 4, SAE) or in a high-fidelity driving simulator running in automated mode (Level 4, SAE). Limited studies assessed motion sickness [2] and simulator sickness [22] when the modality runs in automated mode-and as such generalizations are not yet possible.

\subsection{Motion Sickness and Simulator Sickness}

Motion sickness can occur during or after exposure to AVs [23]. Signs and symptoms associated with motion sickness include nausea, vomiting, drowsiness, sweating, or dizziness [24]. Nausea includes gastrointestinal distress, uncomfortable stomach, excessive sweating, salivation, burping, and/or vomiting. The oculomotor symptoms include headache, eye-strain, difficulty focusing, and blurred vision [25]. Vestibular symptoms include disorientation, dizziness, and vertigo [26]. Individual susceptibility to motion sickness varies by genetic composition (e.g., sex, age) and/or environmental interaction (i.e., passive vs. active participation; [3]). Motion sickness occurs when inhabitants of a motorized vehicle have sensitivity to movement and experience discomfort (e.g., nausea).

Simulator sickness includes similar symptoms to motion sickness [5,27]. The onset of simulator sickness depends on the characteristics of the individual, including the participants' diet [28], the fidelity of the technology-simulator factors such as rendering, refresh rates, visual design, graphics, complexity of the scenarios, number and angle of turns in the scenario-as well as environmental factors such as ambient air temperature. Simulator sickness occurs when the driver experiences the effects of simulated motion while seated stationary in a driving simulator that may result in discomfort (e.g., nausea).

Interestingly, both motion sickness and simulator sickness occur as a result of experiencing incongruence or conflicting stimuli among the kinesthetic, vestibular, and ocular systems $[26,27]$. Although the symptoms of motion and simulator sickness may be similar, the causes are different, as indicated above. Therefore, a better understanding of the self-perceived experience of motion and driving simulator sickness is necessary, if older drivers are to be exposed to these modes of vehicle automation, in order to promote their acceptance and adoption of AVs.

\subsection{Rationale and Significance}

The recent advances in AVs hold promise for important health and safety benefits for older drivers. Although motion sickness and/or simulator sickness may occur among older drivers when exposed to a fully automated shuttle or the driving simulator in automated mode, the phenomenon is currently poorly understood. Moreover, evidence is required to examine some of the contributing factors to make clear determinants of motion and/or simulator sickness in older drivers of various age subgroups (i.e., young-old and old-old) and sex. 


\subsection{Purpose}

The purpose of this study is to quantify the occurrence of motion sickness in an automated shuttle (Level 4, SAE) and the occurrence of simulator sickness in a high-fidelity driving simulator (Level 4, SAE) among older drivers; and to examine the age and sex effects that may contribute to such outcomes.

\subsection{Research Question}

The assumptions are: (1) There may be differences in motion and/or simulator sickness between older drivers exposed to the automated shuttle vs. the automated driving simulator; (2) females (vs. males) may be more susceptible to motion and/or simulator sickness; and (3) those representing the old-old (vs. young-old) may be more susceptible to motion and/or simulator sickness.

The research questions are as follow:

1. Do older drivers experience motion sickness in an automated shuttle (Level 4, SAE) and/or simulator sickness in a high-fidelity driving simulator operating in automated mode (Level 4, SAE)? If they experience motion sickness or simulator sickness, do their reported symptom scores vary?

2. What is the effect of age and sex (if any) on motion and simulator sickness outcomes in this population?

The study findings will address these questions and lead to a better understanding of contributing factors to these occurrences at a time when older drivers consider potential benefits and drawbacks of fully AVs, and make choices related to embracing AVs a mode of transportation.

\section{Materials and Methods}

\subsection{Ethics}

The University of Florida's Institutional Review Board (IRB) approved the study after a full board review (IRB201801988). All participants provided written informed consent prior to their enrolment in this study.

\subsection{Design}

This study is embedded in the parent study, entitled: "UF \& UAB's Phase 1 Demonstration Study: Older Driver Experiences with Autonomous Vehicle Technology" and uses an experimental crossover-repeated measures design [22,29].

\subsection{Recruitment}

Participants were recruited via IRB approved flyers, social media postings, and stakeholder (e.g., retirement communities) referrals. Participants were compensated $\$ 25.00$ for participating in three study visits, each lasting approximately $60 \mathrm{~min}$.

\subsection{Participants}

The study participants were older drivers ( $n=104 ; 65$ years of age or older) who were residents from locations in North Central Florida and surrounding area (e.g., Orlando), with a valid driver's license, and who reported driving within the last six months. Participants excluded from the study were non-English speaking, transportation dependent, or who showed signs of moderate cognitive impairment by scoring $<18$ on the Montreal Cognitive Assessment [30].

\subsection{Setting}

The automated shuttle exposure occurred at a bus depot in Gainesville, FL (100 SE 10th Avenue, Gainesville, FL, USA). The driving simulation exposure occurred in the simulator laboratory, located in the Smart House at Oak Hammock in Gainesville, FL, USA. 


\subsection{Equipment}

\subsubsection{RTI Driving Simulator and Driving Scenario}

The participants were exposed to the Realtime Technologies Inc. (RTI) high-fidelity driving simulator (see Figure 1). Half of the group was exposed to the 5-min acclimation scenario to increase their comfort and adaptation to the driving simulator. The RTI simulator system is installed in a vehicle consisting of seven High Definition (HD) visual channels-three forward channels allowing $180^{\circ}$ field of view on the front, and rear screen for the view on the back, two LCD side mirrors, and one virtual dash display. Graphic resolutions with high fidelity, surrounding audio, and steering wheel button for transitioning into the automated mode are integrated into the simulator. The simulator sickness questionnaire was administered via Qualtrics using an Apple iPad Pro, before and immediately after the exposure to the scenario. The main drive was completed in an automated format in a low to moderate speed $(\leq 15 \mathrm{mph})$ residential and suburban area, with realistic road infrastructure, buildings, and ambient traffic, and lasted 10-min (see Figure 2). The face and content validity of the routes were established using subject-matter experts [29].

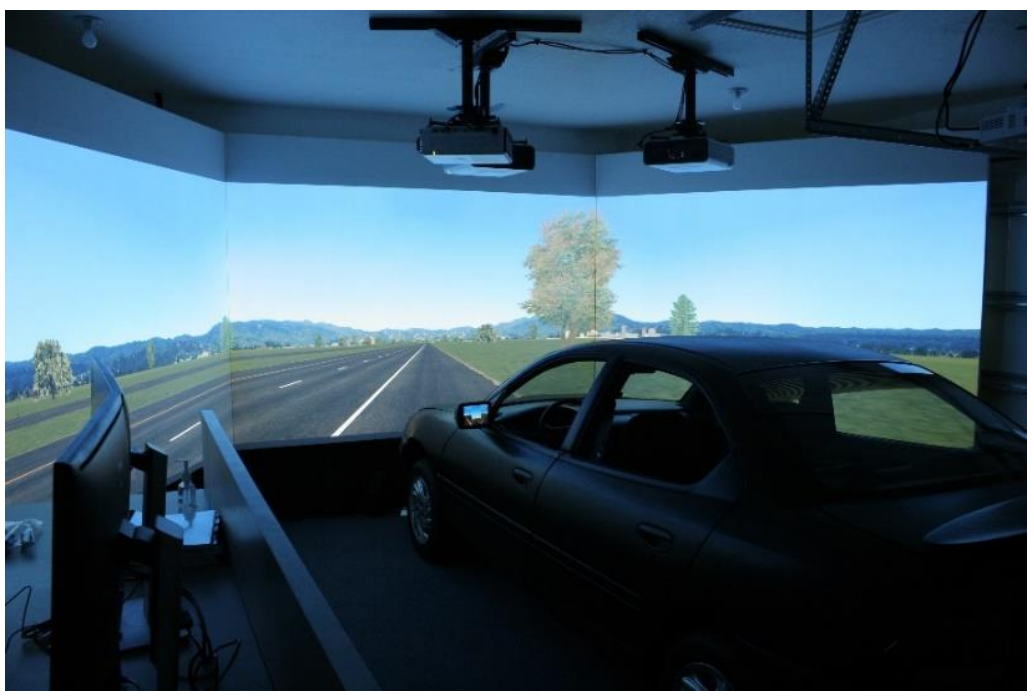

Figure 1. Realtime Technologies Inc. (RTI) High Fidelity Driving Simulator (Level 4, SAE).

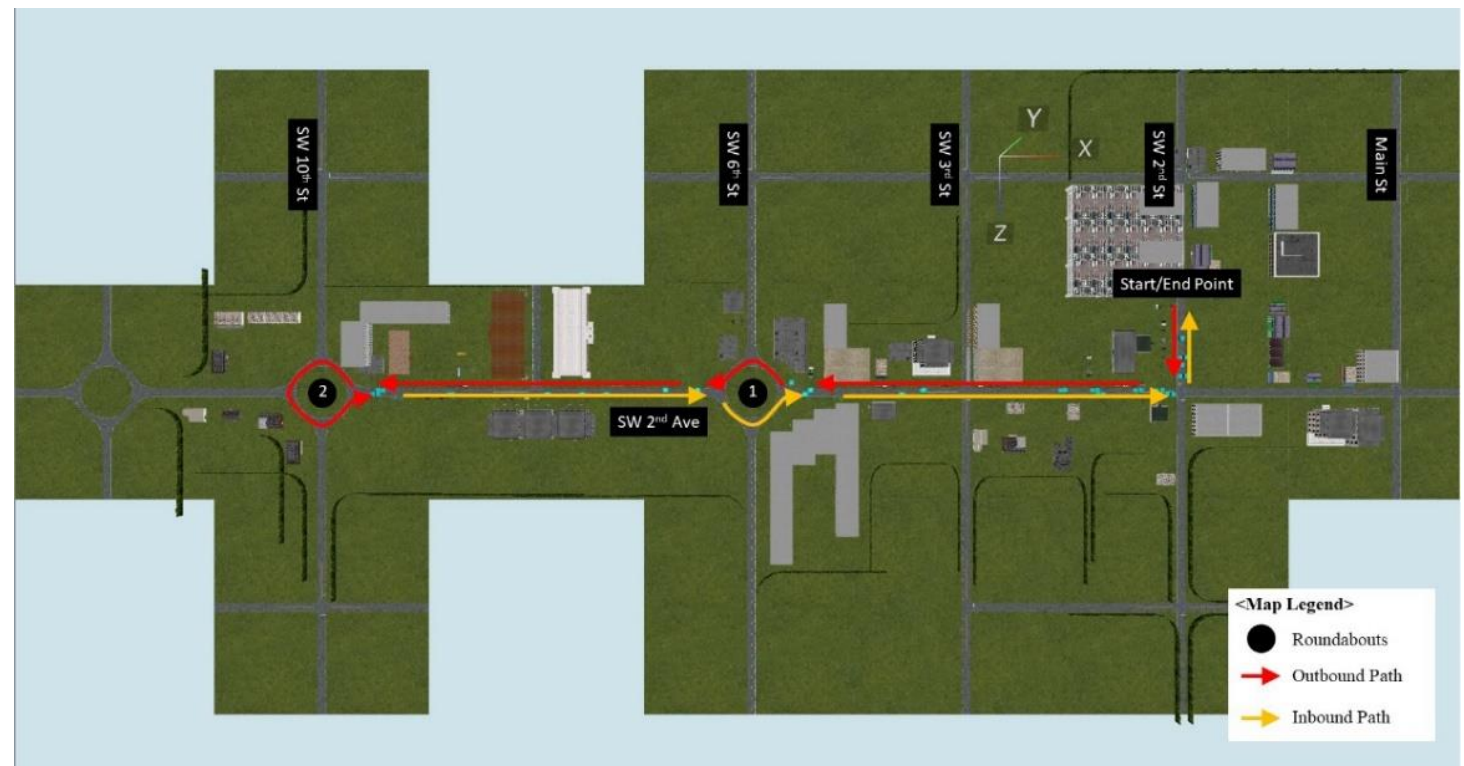

Figure 2. Driving Simulator Route: Ten-minute automated driving simulation (Level 4, SAE) consisted of roundabouts, turns, and stops following the outbound (red) and inbound (yellow) pathways. 


\subsubsection{Automated Shuttle}

The EasyMile shuttle (EZ 10, TransDev North America, Lombard, IL, USA) utilized light detecting sensors to operate autonomously within the specified geographic region (see Figure 3). The number of participants in the vehicle during testing ranged from two to six participants. Although the drive was initially planned to be congruent to the simulator drive, the NHTSA waiver was not yet intact to enroll research participants in the shuttle to run on public roads. As such, an alternative route was planned to continue with the study. The drive occurred in a deserted bus depot, and as such, passengers encountered no traffic, cyclists, or pedestrians (see Figure 4). During segments of the route, the safety operator explained vehicle capabilities and features to the participants. The route lasted roughly $10 \mathrm{~min}$ with the shuttle operating bi-directionally at a low to moderate speed (15-25 mph).

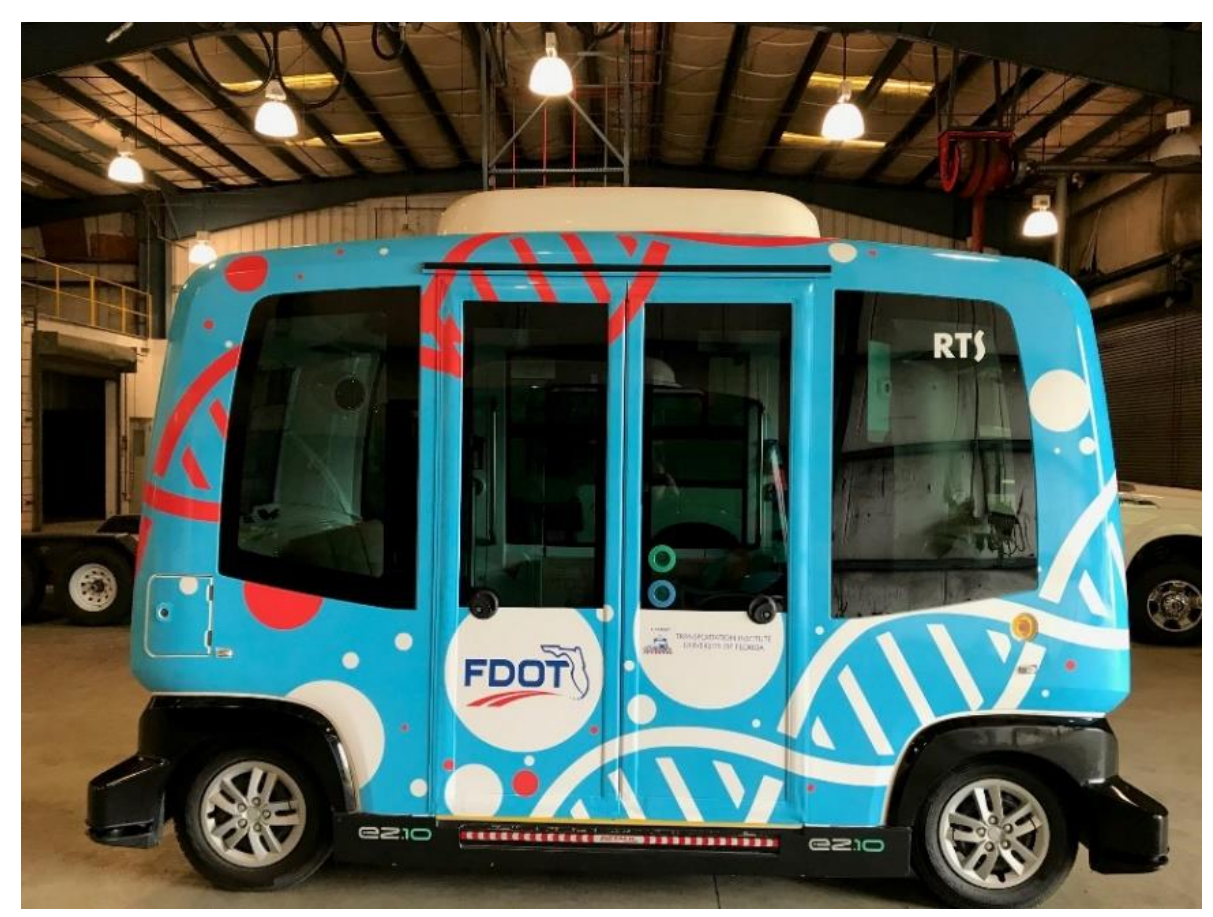

Figure 3. Transdev: EasyMile EZ 10 (Level 4, SAE).

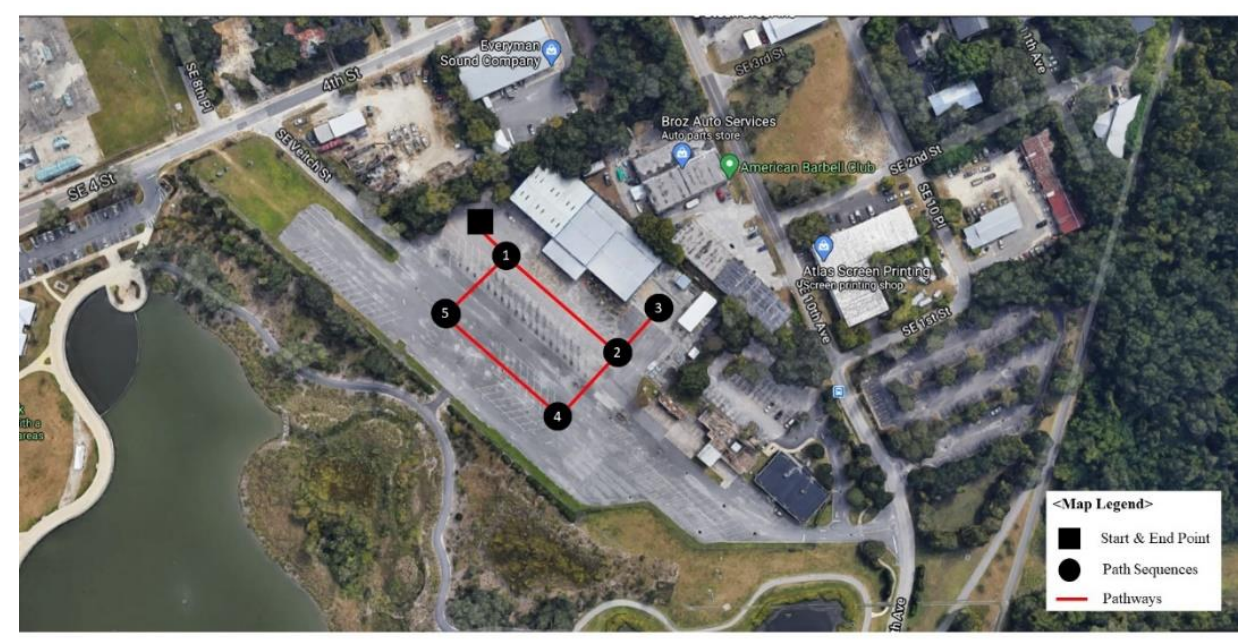

Figure 4. Shuttle Route: Ten-minute automated shuttle ride followed the sequences ( 1 to 5 ) of the pathways (red). 


\subsection{Measures}

A modified version of the demographic and health information form, available through the National Institute of Aging Clinical Research Toolbox, was used to collect age, sex, race, education, relationship, and employment data [31]. The Motion Sickness Assessment Questionnaire (MSAQ), developed and validated for assessing simulator sickness symptoms, was used to assess motion and simulator sickness [10]. The MSAQ consists of 4 items and represents symptom of sweatiness, queasiness, dizziness, and nauseousness, with symptom scores ranging from 0 (not at all) to 10 (severe), collected via self-report on a visual analogue scale.

\subsection{Procedure}

Each participant $(n=104)$ provided written consent and was randomly assigned to be exposed to either the automated shuttle or the driving simulator first, and then crossed over to the other mode. All exposures occurred under good weather conditions during the daytime between the hours of 9 am and $4 \mathrm{pm}$. Participants completed the MSAQ before and after the exposure to the automated shuttle and/or driving simulator.

For the automated shuttle, participants were seated, with the seatbelts fastened, facing either forward or rearward. The safety operator monitored the participants to ensure their comfort and safety, and answered questions pertaining to the ride.

Before the driving simulator exposure, each participant was informed of the simulator sickness protocol to mitigate the occurrence of simulator sickness $[10,28]$. The protocol consisted of offering dietary recommendations prior to the drive (e.g., refrain from consuming alcohol, caffeine, or greasy food, maintain adequate hydration), providing cool comfortable conditions at 72 degrees Fahrenheit during the drive, maintaining air circulation via a fan, and determining and/or managing simulator sickness symptoms [32] shown to be successful in previous older driver studies $[12,22,28]$. Research assistants monitored the participants' comfort and safety during the exposure.

\subsection{Data Collection, Management, and Analysis}

Data were collected and managed through The Research Electronic Data Capture (REDCap) system hosted at the University of Florida [33]. Descriptive inferences included participants' age, sex, race, years of education, relationship status, and current employment status. Continuous variables with normal distribution were presented as mean and standard deviation $(S D)$; non-parametric and categorical variables were displayed as median $(S D)$ and frequencies $(\%)$, respectively. Difference scores were calculated (Post-Pre) using the 4 MSAQ symptom scores (i.e., sweatiness, queasiness, dizziness, nauseousness), and normality was visually inspected via box plots and stem leaf plots. Statistical test, i.e., Shapiro-Wilk, was used to assess the distribution of the data. The Wilcoxon signed-rank test was used to compare participants' motion sickness or simulator sickness between exposure to the different modes. The Mann-Whitney $U$ test was used to assess differences of motion or simulator sickness by age (young-old vs. old-old) and sex (males vs. females) of the participants. All statistical analyses were performed using IBM SPSS, version 26 [34], with a significance level of $\alpha=0.05$, at the $95 \%$ confidence level. Multiple comparisons were adjusted by using the Bonferroni correction $(p=0.05$ divided by the number of related comparisons).

\section{Results}

\subsection{Demographics}

Table 1 summarizes demographic characteristics of the study sample. A total of 104 participants $\left(M_{\mathrm{age}}=74.29, S D_{\mathrm{age}}=5.96 ; 65\right.$ to 91 years $)$ completed the study. About $73 \%$ of participants were college educated and their highest degree was either a doctorate, master's, or bachelor's degree. Normality was violated $\left(p^{\prime} s<0.05\right)$ for all MSAQ difference scores and thus, non-parametric analyses were performed. 
Table 1. Demographics of Older Drivers $(n=104)$.

\begin{tabular}{|c|c|c|}
\hline Factor & Value & Frequency $(\%)$ \\
\hline \multirow{5}{*}{ Age } & 65-69 years old & $25(24 \%)$ \\
\hline & 70-74 years old & $30(30 \%)$ \\
\hline & $75-79$ years old & $24(25 \%)$ \\
\hline & 80-84 years old & $19(18 \%)$ \\
\hline & 85 years and older & $3(3 \%)$ \\
\hline \multirow{2}{*}{ Sex } & Male & $48(46 \%)$ \\
\hline & Female & $56(54 \%)$ \\
\hline \multirow{5}{*}{ Race } & African-American or Black & $8(8 \%)$ \\
\hline & Asian/Pacific Islander & $1(1 \%)$ \\
\hline & Caucasian or White & $92(88 \%)$ \\
\hline & Would rather not say & $1(1 \%)$ \\
\hline & Other & $2(2 \%)$ \\
\hline \multirow{7}{*}{ Education } & High school & $4(4 \%)$ \\
\hline & Some college credits & $10(10 \%)$ \\
\hline & Technical training & $2(2 \%)$ \\
\hline & Associate's degree & $12(11 \%)$ \\
\hline & Bachelor's degree & $21(20 \%)$ \\
\hline & Master's degree & $32(31 \%)$ \\
\hline & $\begin{array}{l}\text { Doctorate/Professional } \\
\text { degree }\end{array}$ & $23(22 \%)$ \\
\hline \multirow{4}{*}{ Relationship } & Single, never married & $6(6 \%)$ \\
\hline & $\begin{array}{l}\text { Married or domestic } \\
\text { partnership }\end{array}$ & $74(71 \%)$ \\
\hline & Widowed & $11(11 \%)$ \\
\hline & Divorced & $13(12 \%)$ \\
\hline \multirow{5}{*}{ Employment } & Part-time & $14(13 \%)$ \\
\hline & Full-time & $5(5 \%)$ \\
\hline & Retired & $83(80 \%)$ \\
\hline & Homemaker & $1(1 \%)$ \\
\hline & Unemployed & $1(1 \%)$ \\
\hline
\end{tabular}

\subsection{Motion or Simulator Sickness Comparison between Automated Shuttle and Driving Simulator Exposures}

The Wilcoxon signed-rank test showed differences in motion or simulator sickness between the automated shuttle and driving simulator exposures. The results revealed that older drivers experienced a statistically significant increase in sickness in all four MSAQ domains, after the driving simulator exposure and when compared, the automated shuttle exposure (Table 2). To control for multiple comparisons, the alpha level was adjusted to $0.0125(0.05 /$ number of related comparisons).

Table 2. Motion and Simulator Sickness Differences between Automated Shuttle and Driving Simulator Exposures $(n=104)$.

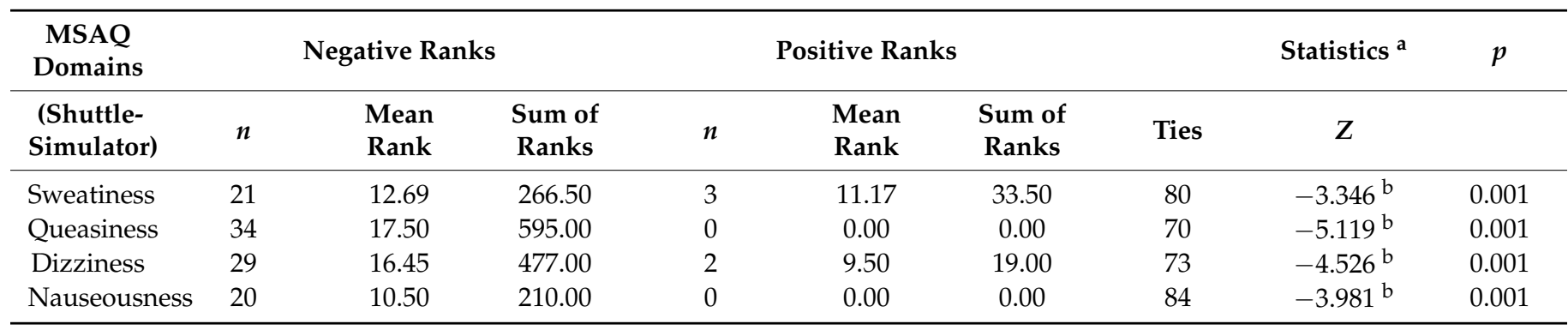

Note: Adjusted alpha level $=0.05 / 4 \leq 0.0125 .^{\mathrm{a}}=$ Wilcoxon Signed Ranks Test. ${ }^{\mathrm{b}}=$ Based on positive ranks. 


\subsection{Motion and Simulator Sickness Comparison by Age and Sex}

The median MSAQ scores were not statistically significantly different between youngold (65-74) and old-old (>75) groups (Table 3). The Mann-Whitney U test displayed differences between males and females for sweatiness in the automated shuttle (Cohen's $\mathrm{d}$ $=0.24, p=0.018$ ) and nauseousness in the driving simulator (Cohen's $\mathrm{d}=0.282, p=0.038$ ). However, these differences were no longer significant after the Bonferroni correction $(p>0.0125)$.

Table 3. Motion and Simulator Sickness Differences between Automated Shuttle and Driving Simulator Groups by Age and Sex.

\begin{tabular}{|c|c|c|c|c|c|c|}
\hline $\begin{array}{c}\text { MSAQ } \\
\text { Domains }\end{array}$ & $\begin{array}{l}\text { Young-Old } \\
\text { Group }\end{array}$ & $\begin{array}{l}\text { Old-Old } \\
\text { Group }\end{array}$ & & Males & Females & \\
\hline & Median (SD) & Median (SD) & $p$ & Median (SD) & Median (SD) & $p$ \\
\hline \multicolumn{7}{|l|}{ Shuttle } \\
\hline Sweatiness & $0.00(0.67)$ & $0.00(1.05)$ & 0.794 & $0.00(0.43)$ & $0.00(1.09)$ & 0.018 \\
\hline Queasiness & $0.00(0.00)$ & $0.00(0.72)$ & 0.280 & $0.00(0.00)$ & $0.00(0.67)$ & 0.355 \\
\hline Dizziness & $0.00(0.00)$ & $0.00(0.00)$ & 1.0 & $0.00(0.00)$ & $0.00(0.00)$ & 1.0 \\
\hline Nauseousness & $0.00(0.00)$ & $0.00(0.00)$ & 1.0 & $0.00(0.00)$ & $0.00(0.00)$ & 1.0 \\
\hline \multicolumn{7}{|l|}{ Simulator } \\
\hline Sweatiness & $0.00(1.89)$ & $0.00(0.97)$ & 0.216 & $0.00(1.70)$ & $0.00(1.42)$ & 0.147 \\
\hline Queasiness & $0.00(1.81)$ & $0.00(1.50)$ & 0.605 & $0.00(1.86)$ & $0.00(1.50)$ & 0.743 \\
\hline Dizziness & $0.00(1.78)$ & $0.00(1.60)$ & 0.553 & $0.00(1.67)$ & $0.00(1.73)$ & 0.884 \\
\hline Nauseousness & $0.00(1.29)$ & $0.00(1.29)$ & 0.038 & $0.00(1.35)$ & $0.00(1.25)$ & 0.680 \\
\hline
\end{tabular}

Note: Adjusted alpha level $=0.05 / 4 \leq 0.0125$. Data are displayed as Median $(S D)$.

\section{Discussion}

This study quantified older drivers' self-reported motion sickness in an automated shuttle and simulator sickness in a driving simulator; and examined the age and sex effects as determinants of the outcomes. Overall, older drivers reported less motion sickness in all four domains (sweatiness, queasiness, dizziness, nauseousness) after the automated shuttle exposure, compared to the driving simulator exposure. Interestingly, we found no age (i.e., young-old vs. old-old) or sex (male vs. female) differences in motion and simulator sickness.

\subsection{Motion and/or Simulator Sickness (Shuttle vs. Driving Simulator)}

The results indicated that older drivers experienced less motion sickness in all four domains after exposure to the automated shuttle (vs. the driving simulator).

Findings of this study align with Sportillo et al.'s study [35], in which they exposed 60 drivers ( 30 males and 30 females, aged 22 to 71years) to a Level-3 (SAE) driving simulator. Level 3 , is considered conditional driving automation, where a human driver is required to "take" the driving task over when requested. They found that $50 \%$ of the drivers experienced simulator sickness. Driving simulation is a valid and reliable research tool to expose older drivers to specific driving conditions without adding undue risk. Findings from this and previous study [22] indicate that driving simulation may offer a safe environment for older drivers to become familiar with, and use of AVs. However, simulator sickness must be mitigated to prevent discomfort and a negative experience of the automated simulated drive, which may, in turn, impact the participants' perceptions of AVs.

No older drivers dropped out from the current study due to motion and/or simulator sickness. The results demonstrated that a majority (Sweatiness: 77\%, Queasiness: 67\%, Dizziness: $70 \%$, Nauseousness: $81 \%$ ) of participants had no differences (i.e., Ties) in the MSAQ scores after exposure to the automated shuttle and driving simulator. This means that a majority of participants did not experience the symptoms; or those who 
did experience symptoms, could adequately manage to ensure study completion. This finding differs from other studies that had higher percentages $(16.7 \%$ [10]; 30\% [36]) of dropout because of simulator sickness. Zero attrition rate may be an indicator that the symptoms were tolerable. As such, we discern that the automated shuttle and the driving simulator may be beneficial for exposing older drivers to AV technology. Likewise, it seems, compared to other literature $[10,36]$ that this study's protocol and mitigation strategies could have benefitted the drivers to at least curtail the severity of symptoms for those who have experienced such symptoms.

\subsection{Motion and/or Simulator Sickness by Age (Young-Old vs. Old-Old) and Sex (Male vs. Female)}

Contrary to previous studies, the current study found no differences when comparing MSAQ scores between age groups (i.e., young-old and old-old). In Brooks et al. [10] and Keshavarz et al. studies [37], age effects existed with older adults showing a statistically significant increase (Brooks et al., Cohen's d $=0.91$; Keshavarz et al., Cohen's $d=0.67$ ) in simulator sickness scores compared to younger adults after exposure to a driving simulator. The difference in findings between studies may be due to Brooks et al. and Keshavarz et al. utilizing a younger driver comparison group, whereas the current study compared young-old and old-old drivers. Moreover, other studies report no difference in simulator sickness between older and younger adults [38], or among younger, middle-aged, and older adults [39], which aligns with the findings of this current study.

Earlier studies suggest that females are more susceptible to experiencing motion sickness compared to their male counterparts [40-42]. However, in this study, the researchers found no differences in the MSAQ scores between male and female participants. Similarly, Mourant et al. [43] reported no differences in simulator sickness scores between males and females (Mann-Whitney U Test, $p=0.56$ ). Additionally, consistent with our study's results, Sportillo et al., [35] and Keshavarz et al., [44] confirm that simulator sickness scores are not statistically significantly different in female vs. male participants. Based on this current study's results, we also see that the finding is holding true for sex comparisons in automated vehicles.

\subsection{Limitations, Strengths, and Future Studies}

The MSAQ, is a self-report measure, and therefore may infuse bias to identify motion and/or simulator sickness. More objective and physiological measures e.g., galvanic skin responses to measure "sweatiness", or heart rate variability to measure "queasiness" may allow for more rigorous comparisons in addition to the MSAQ. This study had a large sample size, and is one of the first to compare motion and simulator sickness via automated shuttle and driving simulator exposure (Level 4, SAE). The zero percent attrition indicates that the findings of this study support the use of an automated shuttle and driving simulator for exposing older drivers to such technologies.

The next step for this study is to compare drivers across the lifespan (i.e., younger and middle-aged drivers) or populations with special needs (e.g., those with mild cognitive impairment) to older drivers. These meaningful comparisons will further reveal the riskbenefit characteristics of exposing different populations to the two modes (i.e., automated shuttle and automated driving simulator; Level 4, SAE) of these automated technologies.

\section{Conclusions}

The results of this study suggest that older drivers who are exposed to the automated driving simulator show a statistically significant increase in simulator sickness symptoms across the four domains (sweatiness, queasiness, dizziness, nauseousness) compared to the same group being tested in the automated shuttle. No age and sex differences were detected within the groups, and no older drivers dropped out of the study due to motion or simulator sickness. As such, the automated shuttle and driving simulator hold great promise for continued exposure of older drivers to $\mathrm{AV}$ technologies when carefully designed motion or simulator sickness protocols are in place. 
Author Contributions: Conceptualization, S.C. and V.P.S.; Methodology, S.C., J.M., J.W., J.R. and V.P.S.; Software, J.R.; Validation; S.C., V.P.S. and J.M.; Formal Analysis; J.M. and S.W.H.; Investigation, J.M., J.W. and S.W.H.; Resources, S.C., J.M., J.W. and S.W.H.; Data Curation, J.M., J.R., J.W. and S.W.H.; Writing, S.C., J.M., S.W.H., J.W., J.R. and V.P.S.; Writing-Review \& Editing, S.C., J.M., S.W.H., J.W., J.R. and V.P.S.; Visualization, S.C., J.M., J.R. and S.W.H.; Supervision, S.C.; Project Administration, S.C.; Funding Acquisition, S.C. All authors have read and agreed to the published version of the manuscript.

Funding: This research was funded by the Southeastern Transportation Research, Innovation, Development, and Education (STRIDE) Center (Project D2, \#69A3551747104). The APC was waived by the invitation of the Safety journal special issue.

Institutional Review Board Statement: The study was conducted according to the guidelines of the Declaration of Helsinki, and approved by the Institutional Review Board of the University of Florida (IRB201801988 and 8/22/2018).

Informed Consent Statement: Written informed consent was obtained from all subjects involved in the study.

Data Availability Statement: The data presented in this study are available upon request.

Acknowledgments: The authors wish to acknowledge the Institute for Mobility, Activity and Participation at the University of Florida which provided the infrastructure and support for this study. The TREND Lab at the University of Alabama at Birmingham also provided support for this study.

Conflicts of Interest: The authors declare no conflict of interest. The sponsors had no role in the design, execution, interpretation, or writing of the study.

\section{References}

1. Pettigrew, S. Why public health should embrace the autonomous car. Aust. N. Z. J. Public Health 2016, 41, 5-7. [CrossRef]

2. Jones, M.L.H.; Le, V.C.; Ebert, S.M.; Sienko, K.H.; Reed, M.P.; Sayer, J.R. Motion sickness in passenger vehicles during test track operations. Ergonomics 2019, 62, 1357-1371. [CrossRef] [PubMed]

3. Golding, J.F. Motion sickness susceptibility. Auton. Neurosci. 2006, 129, 67-76. [CrossRef] [PubMed]

4. Stephanidis, C. Universal Access in Human-Computer Interaction. Context Diversity. In Proceedings of the 6th International Conference, UAHCI 2011, Held as Part of HCI International 2011, Orlando, FL, USA, 9-14 July 2011; Springer Science \& Business Media: Berlin/Heidelberg, Germany, 2011.

5. Keshavarz, B.; Hecht, H.; Lawson, B.D. Visually induced motion sickness: Characteristics, causes, and countermeasures. In Handbook of Virtual Environments: Design, Implementation, and Applications, 2nd ed.; Hale, K.S., Stanney, K.M., Eds.; CRC Press Taylor \& Francis Group: Boca Raton, FL, USA, 2014; pp. 648-697.

6. Koohestani, A.; Nahavandi, D.; Asadi, H.; Kebria, P.M.; Khosravi, A.; Alizadehsani, R.; Nahavandi, S. A knowledge dis-covery in motion sickness: A comprehensive literature review. IEEE Access 2019, 7, 85755-85770. [CrossRef]

7. Mullen, N.W.; Weaver, B.; Riendeau, J.A.; Morrison, L.E.; Bédard, M. Driving Performance and Susceptibility to Simulator Sickness: Are They Related? Am. J. Occup. Ther. 2010, 64, 288-295. [CrossRef]

8. Turner, M. Motion sickness in public road transport: Passenger behaviour and susceptibility. Ergonomics 1999, 42, 444-461. [CrossRef]

9. Koch, A.; Cascorbi, I.; Westhofen, M.; Dafotakis, M.; Klapa, S.; Kuhtz-Buschbeck, J.P. The Neurophysiology and Treatment of Motion Sickness. Dtsch. Aerzteblatt Online 2018, 115, 687-696. [CrossRef]

10. Brooks, J.O.; Goodenough, R.R.; Crisler, M.C.; Klein, N.D.; Alley, R.L.; Koon, B.L.; Logan, W.C.; Ogle, J.H.; Tyrrell, R.A.; Wills, R.F. Simulator sickness during driving simulation studies. Accid. Anal. Prev. 2010, 42, 788-796. [CrossRef]

11. Reinhard, R.; Rutrecht, H.M.; Hengstenberg, P.; Tutulmaz, E.; Geissler, B.; Hecht, H.; Muttray, A. The best way to assess visually induced motion sickness in a fixed-base driving simulator. Transp. Res. Part F Traffic Psychol. Behav. 2017, 48, 74-88. [CrossRef]

12. Shechtman, O.; Classen, S.; Stephens, B.; Bendixen, R.; Belchior, P.; Sandhu, M.; McCarthy, D.; Mann, W.; Davis, E. The Impact of Intersection Design on Simulated Driving Performance of Young and Senior Adults. Traffic Inj. Prev. 2007, 8, 78-86. [CrossRef]

13. Kandasamy, D.; Betz, M.E.; DiGuiseppi, C.; Mielenz, T.P.; Eby, D.W.; Molnar, L.J.; Hill, L.; Strogatz, D.; Li, G. Self-reported health conditions and related driving reduction in older drivers. Occup. Ther. Health Care 2018, 32, 363-379. [CrossRef] [PubMed]

14. Sanford, S.; Rapoport, M.J.; Tuokko, H.; Crizzle, A.; Hatzifilalithis, S.; Laberge, S. Canadian Consortium on Neuro-degeneration in Aging Driving and Dementia Team. Independence, loss, and social identity: Perspectives on driving cessation and dementia. Dementia 2019, 18, 2906-2924. [CrossRef] 
15. Edwards, J.D.; Lunsman, M.; Perkins, M.; Rebok, G.W.; Roth, D.L. Driving Cessation and Health Trajectories in Older Adults. J. Gerontol. Ser. A Biomed. Sci. Med. Sci. 2009, 64, 1290-1295. [CrossRef] [PubMed]

16. Marottoli, R.A.; de Leon, C.F.M.; Glass, T.A.; Williams, C.S.; Cooney, L.M., Jr.; Berkman, L.F. Consequences of driving cessation: Decreased out-of-home activity levels. J. Gerontol. Ser. B Psychol. Sci. Soc. Sci. 2000, 55, S334-S340. [CrossRef] [PubMed]

17. Musselwhite, C. The importance of driving for older people and how the pain of driving cessation can be reduced. Signpost 2011, $15,22-26$.

18. Society of Automotive Engineers International. Taxonomy and Definitions for Terms Related to Driving Automation Systems for On-road Motor Vehicles (J3016_201806); Society of Automotive Engineers International: Warrendale, PA, USA, 2018.

19. Anderson, J.M.; Nidhi, K.; Stanley, K.D.; Sorensen, P.; Samaras, C.; Oluwatola, O.A. Autonomous Vehicle Technology: A Guide for Policymakers; Rand Corporation: Santa Monica, CA, USA, 2014.

20. Cicchino, J.B.; McCartt, A.T. Trends in older driver crash involvement rates and survivability in the United States: An update. Accid. Anal. Prev. 2014, 72, 44-54. [CrossRef] [PubMed]

21. Moorthy, A.; De Kleine, R.; Keoleian, G.; Good, J.; Lewis, G. Shared Autonomous Vehicles as a Sustainable Solution to the Last Mile Problem: A Case Study of Ann Arbor-Detroit Area. SAE Int. J. Passeng. Cars Electron. Electr. Syst. 2017, 10, 328-336. [CrossRef]

22. Classen, S.; Mason, J.; Wersal, J.; Sisiopiku, V.; Rogers, J. Older Drivers' Experience with Automated Vehicle Technology: Interim Analysis of a Demonstration Study. Front. Sustain. Cities 2020, 2. [CrossRef]

23. Yusof, N.M.; Karjanto, J.; Kapoor, S.; Terken, J.; Delbressine, F.; Rauterberg, M. Experimental setup of motion sickness and situation awareness in automated vehicle riding experience. In Proceedings of the 9th International Conference on Auto-motive User Interfaces and Interactive Vehicular Applications Adjunct, Oldenburg, Germany, 24-27 September 2017; pp. 104-109.

24. Graybiel, A.; Wood, C.D.; Miller, E.F.; Cramer, D.B. Diagnostic Criteria for Grading the Severity of Acute Motion Sickness; Naval Aerospace Medical Institute, Naval Aerospace Medical Center: Pensacola, FL, USA, 1968; Volume 39, pp. $453-455$.

25. LaViola, J.J., Jr. A discussion of cybersickness in virtual environments. Assoc. Comput. Mach. Sigchi Bull. 2000, 32, 47-56. [CrossRef]

26. Kennedy, R.S.; Lane, N.E.; Berbaum, K.S.; Lilienthal, M.G. Simulator Sickness Questionnaire: An Enhanced Method for Quantifying Simulator Sickness. Int. J. Aviat. Psychol. 1993, 3, 203-220. [CrossRef]

27. Kennedy, R.S.; Drexler, J. Research in visually induced motion sickness. Appl. Ergon. 2010, 41, 494-503. [CrossRef] [PubMed]

28. Classen, S.; Bewernitz, M.; Shechtman, O. Driving Simulator Sickness: An Evidence-Based Review of the Literature. Am. J. Occup. Ther. 2011, 65, 179-188. [CrossRef] [PubMed]

29. Classen, S.; Wersal, J.; Mason, J.; Rogers, J.; Sisiopiku, V. Face and Content Validity of an Automated Vehicle Road Course and a Corresponding Simulation Scenario. Front. Futur. Transp. 2020, 1. [CrossRef]

30. Nasreddine, Z.S.; Phillips, N.A.; Bedirian, V.; Charbonneau, S.; Whitehead, V.; Collin, I.; Cummings, J.L.; Chertkow, H. The Montreal Cognitive Assessment, MoCA: A Brief Screening Tool for Mild Cognitive Impairment. J. Am. Geriatr. Soc. 2005, 53, 695-699. [CrossRef]

31. US Department of Health \& Human Services. Clinical Research Study Investigator's Toolbox. Available online: https://www.nia. nih.gov/research/clinical-research-study-investigators-toolbox\#forms (accessed on 1 January 2021).

32. Stern, E.B.; Akinwuntan, A.E.; Hirsch, P. Simulator sickness: Strategies for mitigation and prevention. In Driving Simulation for Assessment, Intervention, and Training: A Guide for Occupational Therapy and Health Care Professionals, 1st ed.; Classen, S., Ed.; American Occupational Therapy Press: North Bethesda, MD, USA, 2017; pp. 107-120.

33. Harris, P.A.; Taylor, R.; Minor, B.L.; Elliott, V.; Fernandez, M.; O’Neal, L.; McLeod, L.; Delacqua, G.; Delacqua, F.; Kirby, J.; et al. The REDCap consortium: Building an international community of software platform partners. J. Biomed. Inform. 2019, 95, 103208. [CrossRef] [PubMed]

34. George, D.; Mallery, P. IBM SPSS Statistics 26 Step by Step: A Simple Guide and Reference, 16th ed.; Routledge: New York, NY, USA, 2019.

35. Sportillo, D.; Paljic, A.; Ojeda, L. Get ready for automated driving using Virtual Reality. Accid. Anal. Prev. 2018, 118, 102-113. [CrossRef] [PubMed]

36. Jacobs, M.; Van Der Zwaan, K.F.; Hart, E.P.; Groeneveld, G.J.; Roos, R.A. Comparable rates of simulator sickness in Huntington's disease and healthy individuals. Transp. Res. Part F Traffic Psychol. Behav. 2019, 60, 499-504. [CrossRef]

37. Keshavarz, B.; Novak, A.C.; Hettinger, L.J.; Stoffregen, T.A.; Campos, J.L. Passive restraint reduces visually induced motion sickness in older adults. J. Exp. Psychol. Appl. 2017, 23, 85-99. [CrossRef]

38. Saryazdi, R.; Bak, K.; Campos, J.L. Inattentional Blindness During Driving in Younger and Older Adults. Front. Psychol. 2019, 10, 880. [CrossRef] [PubMed]

39. Domeyer, J.E.; Cassavaugh, N.D.; Backs, R.W. The use of adaptation to reduce simulator sickness in driving assessment and research. Accid. Anal. Prev. 2013, 53, 127-132. [CrossRef]

40. Diels, C.; Bos, J.E. Self-driving carsickness. Appl. Ergon. 2016, 53, 374-382. [CrossRef] [PubMed] 
41. Iskander, J.; Attia, M.; Saleh, K.; Nahavandi, D.; Abobakr, A.; Mohamed, S.; Asadi, H.; Khosravi, A.; Lim, C.P.; Hossny, M. From car sickness to autonomous car sickness: A review. Transp. Res. Part F Traffic Psychol. Behav. 2019, 62, 716-726. [CrossRef]

42. Paillard, A.C.; Quarck, G.; Paolino, F.; Denise, P.; Paolino, M.; Golding, J.F.; Ghulyan-Bedikian, V. Motion sickness susceptibility in healthy subjects and vestibular patients: Effects of gender, age and trait-anxiety. J. Vestib. Res. 2013, 23, 203-209. [CrossRef] [PubMed]

43. Mourant, R.R.; Rengarajan, P.; Cox, D.; Lin, Y.; Jaeger, B.K. The effect of driving environments on simulator sickness. In Proceedings of the Human Factors and Ergonomics Society Annual Meeting, Baltimore, MD, USA, 1-5 October 2007; Sage CA: Los Angeles, CA, USA, 2007; Volume 51, pp. 1232-1236.

44. Keshavarz, B.; Ramkhalawansingh, R.; Haycock, B.; Shahab, S.; Campos, J. Comparing simulator sickness in younger and older adults during simulated driving under different multisensory conditions. Transp. Res. Part F Traffic Psychol. Behav. 2018, 54, 47-62. [CrossRef] 